\title{
Ligand efficiency metrics: why all the fuss?
}

\author{
"The best argument for ligand efficiency is simply the assertion that \\ on average smaller drugs have lower cost of goods, tend to be more soluble \\ and bioavailable, and have fewer metabolic liabilities."
}

Keywords: drug discovery metrics $\bullet$ fit quality $\bullet$ ligand efficiency $\bullet$ lipophilic ligand efficiency

Ligand efficiency (LE) is most commonly defined as the ratio of the affinity of a ligand divided by the number of heavy (nonhydrogen) atoms in the molecule (LE $=\Delta \mathrm{G} /[$ number of heavy atoms $])$. This ratio was first described in 1999 [1] and gained widespread popularity in drug discovery circles shortly thereafter [2]. The rapid uptake of LE in drug discovery was likely facilitated by the already widespread use of Lipinski's rules (another size restraining metric) [3] and a burgeoning interest in fragment-based approaches to drug discovery [4]. Ligand efficiency validated fragment-based design and offered a simple measure to keep molecular size in check while undertaking a lead optimization program. Like the rule of five, the rapid adoption of LE by medicinal chemists was probably aided by the simplicity of the metric (no need for complicated algorithms) and the fact that it neatly captured a compelling concept: namely that it is in general better to bring drug discovery candidates forward that are small rather than large. Indeed, many of the productivity problems plaguing the industry are sometimes ascribed to the growing number of potent 'rocks' being produced by discovery efforts driven by in vitro biology [5]. Simple metrics, like LE or the rule of five, help bring the problem of molecular size inflation into focus, in a way that is easy to understand.

Ligand efficiency has been reviewed recently [6], and the reader is referred to that paper for a detailed description of its many flavors (e.g., LE and lipophilic ligand efficiency [LLE]). The review describes the most common efficiency measures in detail, provides references to examples of their application and compares the advantages and disadvantages of different metrics.

\section{Concerns about validity}

Recently, there has been a backlash of sorts against ligand efficiency metrics in the literature, which has in some cases been pointed. Titles such as 'Ligand efficiency metrics considered harmful', [7] and 'Improving the plausibility of success with inefficient metrics', [8] leave little doubt where the authors stand. Similarly there have been a number of volleys back and forth in prominent drug discovery blogs $[9,10]$. Indeed these critical articles, and blog entries, purport to shoot all manner of theoretical and/or mathematical holes in ligand efficiency as a concept. Some of the objections are valid up to a point: there are examples of drugs with poor LE; LE does not account for differences in atom types or functionality; LE has scaling issues and represents an imprecise tool for normalizing potency; and a number of metrics have evolved from LE, a situation that may cause confusion in the medicinal chemistry community. To be fair, some of these shortcomings are the reason for new ligand efficiency metrics such as LLE [11] and fit quality (FQ) [12]. Some of the more visceral negative responses to ligand efficiency metrics may have something to do with the way they are sometimes applied in managing drug discovery programs. There are at least anecdotal examples of managers, or even companies, employing LE and other metrics as hard and fast limits for compound progression. The rigid applica-

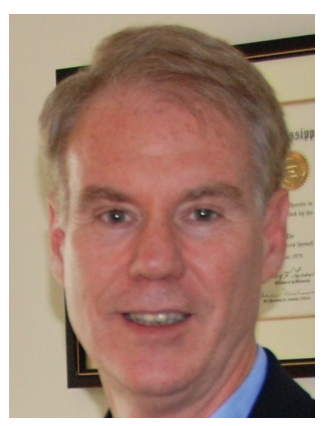

Charles H Reynolds Gfree Bio, LLC, 3805 Old Easton Road, Doylestown, PA 18902, USA creynolds@gfreebio.com 
tion of these 'metrics' in stage-gate decisions does, of course, create concern since there are myriad complex considerations that go into the development of a drug candidate. While benchmarks such as LE, might be convenient discussion points, they are very blunt instruments if instituted as immutable requirements.

The theoretical and/or mathematical arguments used to discredit ligand efficiency represent, in my view, misguided attempts to poke holes in a very simple rule of thumb, using complex and in some cases inaccurate, or inapplicable, mathematical, thermodynamic or other arguments. LE is just an easy to calculate, easy to understand, rule of thumb. In a recent article, LE was compared with fuel economy (miles/gallon) [13]. Few would use miles per gallon as their only criterion for choosing an automobile, but it is certainly useful if one is attempting to find a vehicle that is as efficient as possible, whether motorcycle or large truck. Using an alternative financial analogy: heavy atoms are the currency we spend to achieve high-affinity binding, and all else being equal it is better to spend fewer for a given potency. In the end, the value of ligand efficiency can only be evaluated by its usefulness in thinking about medicinal chemistry problems, it is not a fundamental property.

\section{Application of ligand efficiency measures}

With all the noise, the average medicinal chemist can be forgiven for being confused with regard to whether, or how, ligand efficiency metrics might be used. Indeed, some may be reluctant to use them at all for fear of violating some fundamental physical law. Such concerns are misplaced. Ligand efficiency metrics have been widely shown to have great utility as guideposts that provide a quick measure of how effective a given compound is relative to its size against a given target.

"...it is important to keep ligand efficiency metrics in perspective for what they are: simple, but often useful, tools for ligand design.”

If we consider the most widely used metric, LE, there are a number of general considerations to keep in mind. First, LE can break down when comparing ligands of disparate size (LLE, FQ and size independent ligand efficiency [SILE] are better) [6-8,11-12]. But as long as one is aware of this factor, it really does not prevent LE from being used for small or large ligands. The value of LE or LLE, that is attainable, is ultimately dictated to a large degree by the size and characteristics of the target [14]. A drug candidate for a large binding site (e.g., protein-protein or protease inhibitor) will by the nature of the target likely have lower LE than candidates for targets with smaller binding sites. It is important to be familiar with these limitations, and keep in mind what the different metrics measure with regard to potency and molecular size. But when used pragmatically, they can help chemists and biologists alike evaluate screening hits, leads or even clinical candidates in a way that looks beyond affinity. Using ligand efficiency metrics as strict stage-gate criteria is problematic because of these target-based differences and the simple fact that it is often necessary at some point to add hydrophobic bulk to achieve sufficient potency.

\section{Some convenient efficiency metrics}

It is important for medicinal chemists to be familiar with at least the most common ligand efficiency metrics and how they behave differently. The best known quantity ( $\mathrm{LE}=$ affinity/HA) is a convenient way to represent potency per atom when evaluating ligand binding. LE is a perfectly valid expression and has been used extensively in many drug discovery programs. One should be aware of the nonlinear scaling of LE over large size ranges. Lipophilic ligand efficiency (LLE = DG- $\log \mathrm{P})$ is an alternative efficiency metric that uses $\log \mathrm{P}$ as the normalizing quantity rather than number of atoms. This provides better scaling over wider size ranges and has the advantage of normalizing potency relative to a property of the ligand $(\log \mathrm{P})$, that has a long history of being employed in drug discovery and is familiar to most medicinal chemists. The explicitly scaled ligand efficiency metrics are the quantities like FQ [12] and SILE [15]. These metrics produce normalized scores across a diverse range of molecular sizes. For example, in the case of $\mathrm{FQ}$, the binned scaling factors have been chosen so that efficient ligands have scores $(F Q=$ LE/LE_scaled) near one regardless of size. This consistency can be helpful for some tasks, such as evaluation of screening hits [16]. These and other efficiency metrics that have been well described in the literature [6] can be valuable in many drug discovery contexts.

\section{Conclusion}

There is no need to become overly concerned with noisy arguments for or against ligand efficiency metrics being exchanged in the literature. There is no fundamental theoretical or mathematical objection that invalidates, or for that matter justifies, these metrics. The best argument for ligand efficiency is simply the assertion that on average smaller drugs have lower cost of goods, tend to be more soluble and bioavailable, and have fewer metabolic liabilities. The 'history' of many targets supports this view since newer generations of drugs are in many cases more potent, smaller in size, or 
both. For example, the first HIV-1 protease inhibitor, saquinavir $(\mathrm{LE}=0.25)$, is much larger and less potent than darunavir $(\mathrm{LE}=0.40)$. This evolution is a result of continuous efforts to hone potency and other desirable molecular properties. A recent concrete example of a drug discovery success that was very much driven by a concern for ligand efficiency is the BACE inhibitor currently in clinical trials [17]. Finally, it is important to keep ligand efficiency metrics in perspective for what they are: simple, but often useful, tools for ligand design. A 2010 paper by Perola [18] is recommended for its sensible insights into the application of efficiency metrics in discovery.

\section{References}

1 Kuntz ID, Chen K, Sharp KA, Kollman PA. The maximal affinity of ligands. Proc. Natl Acad. Sci. USA 96(18), 9997-10002 (1999).

2 Hopkins Andrew L, Groom Colin R, Alex A. Ligand efficiency: a useful metric for lead selection. Drug Discov. Today 9(10), 430-431 (2004).

3 Lipinski CA. Lead- and drug-like compounds: the rule-of-five revolution. Drug Discov. Today Technol. 1(4), 337-341 (2004).

4 Rees DC, Congreve M, Murray CW, Carr R. Fragment-based lead discovery. Nat. Rev. Drug Discov. 3(8), 660-672 (2004).

5 Rishton GM. Nonleadlikeness and leadlikeness in biochemical screening. Drug Discov. Today 8(2), 86-96 (2003).

6 Hopkins AL, Keseru GM, Leeson PD, Rees DC, Reynolds $\mathrm{CH}$. The role of ligand efficiency metrics in drug discovery. Nat. Rev. Drug Discov. 13(2), 105-121 (2014).

7 Kenny P, Leitão A, Montanari C. Ligand efficiency metrics considered harmful. J. Comput. Aided Mol. Des. 28(7) 699-710 (2014).

8 Shultz MD. Improving the plausibility of success with inefficient metrics. ACS Med. Chem. Lett. 5(1), 2-5 (2013).

9 Ligand efficiency: a response to Shultz. http://pipeline.corante.com

\section{Acknowledgements}

The author thanks P Bernstein and C Murray for reading a draft of this manuscript and providing many useful comments.

\section{Financial \& competing interests disclosure}

The author has no relevant affiliations or financial involvement with any organization or entity with a financial interest in or financial conflict with the subject matter or materials discussed in the manuscript. This includes employment, consultancies, honoraria, stock ownership or options, expert testimony, grants or patents received or pending, or royalties.

No writing assistance was utilized in the production of this manuscript.

10 Erlanson, D. In defense of ligand efficiency - and poll. http://practicalfragments.blogspot.com

11 Leeson PD, Springthorpe B. The influence of drug-like concepts on decision-making in medicinal chemistry. Nat. Rev. Drug Discov. 6(11) 881-890 (2007).

12 Reynolds $\mathrm{CH}$, Tounge BA, Bembenek SD. Ligand binding efficiency: trends, physical basis, and implications. J. Med. Chem. 51(8), 2432-2438 (2008).

13 Murray CW, Erlanson DA, Hopkins AL et al. Validity of ligand efficiency metrics. ACS Med. Chem. Lett. 5(6), 616-618 (2014).

14 Vieth M, Sutherland JJ. Dependence of molecular properties on proteomic family for marketed oral drugs. J. Med. Chem. 49, 3451-3453 (2006).

15 Nissink JW. Simple size-independent measure of ligand efficiency. J. Chem. Inf. Model. 49(6), 1617-1622 (2009).

16 Zhu T, Cao S, Su P-C et al. Hit identification and optimization in virtual screening: practical recommendations based on a critical literature analysis: miniperspective. J. Med. Chem. 56(17), 6560-6572 (2013).

17 Stamford AW, Scott JD, Li SW et al. Discovery of an orally available, brain penetrant BACE1 inhibitor that affords robust CNS abeta reduction. ACS Med. Chem. Lett. 3(11), 897-902 (2012).

18 Perola E. An analysis of the binding efficiencies of drugs and their leads in successful drug discovery programs. J. Med. Chem. 53(7), 2986-2997 (2010). 